\title{
Auxiliary soft beam for the amplification of the elasto-capillary coiling: towards stretchable electronics
}

\author{
Paul Grandgeorge ${ }^{\mathrm{a}}$, Arnaud Antkowiak ${ }^{\mathrm{a}, \mathrm{b}}$, Sébastien Neukirch ${ }^{\mathrm{a}, *}$ \\ ${ }^{a}$ Institut Jean Le Rond d'Alembert, Sorbonne Universités, UPMC Univ. Paris 06, CNRS, UMR 7190, 75005 Paris, France \\ ${ }^{b}$ Surface du Verre et Interfaces, UMR 125 CNRS/Saint-Gobain, F-93303 Aubervilliers, France
}

\begin{abstract}
A flexible fiber carrying a liquid drop may coil inside the drop thereby creating a drop-on-fiber system with an ultraextensible behaviour. During compression, the excess fiber is spooled inside the droplet and capillary forces keep the system taut. During subsequent elongation, the fiber is gradually released and if a large number of spools is uncoiled a high stretchability is achieved. This mechanical behaviour is of interest for stretchable connectors but information, may it be electronic or photonic, usually travels through stiff functional materials. These high Young's moduli, leading to large bending rigidity, prevent in-drop coiling. Here we overcome this limitation by attaching a beam of soft elastomer to the functional fiber, thereby creating a composite system which exhibits in-drop coiling and carries information while being ultra-extensible. We present a simple model to explain the underlying mechanics of the addition of the soft beam and we show how it favors in-drop coiling. We illustrate the method with a two-centimeter long micronic PEDOT:PSS conductive fiber joined to a PVS soft beam, showing the system conveys electricity throughout a $1900 \%$ elongation.
\end{abstract}

Keywords: capillarity, stretchable electronics, buckling, composite materials

\section{Introduction}

At small scales capillary forces may be used to fold elastic structures, and several studies have shown how one can design shape and form in microstructures by the careful 5 utilization of surface tension, provided Young's moduli are low enough or structures are thin enough [1, 2, 3, 4, 5. The mechanics and physics of drops on fibers has been studied from the point of view of wettability and mist capture 6. 7, 8, 9] but recently, finding inspiration in spider webs [10, 11, 12, we have seen the introduction of elastocapillary drop-on-fiber systems where the flexible fiber coils inside a liquid drop. This spooling mechanism provides the system with a constant force behavior under extension (i.e. vanishing stretching modulus) in addition to extreme 15 extensibility [2, 13, 14, 15. In-drop coiling basically requires thin fibers and up to now $\sim 10 \mu \mathrm{m}$ diameters and $\sim 10 \mathrm{MPa}$ materials have been successfully used. With the aim to design functional microstructures utilizing indrop coiling, we turn to the use of metalic materials with 20 the drawback that functional materials usually have high Young's moduli [16] (metals for electronics, glass for photonics, for which $E \sim 50-100 \mathrm{GPa}$ ). For these materials, if the bending rigidity is to be kept low, the structure has to be dramatically thin, in the order of nanometers. Yet

\footnotetext{
* Corresponding author

Email address: sebastien.neukirch@upmc.fr (Sébastien Neukirch)
}

lose their functionality (electric resistance of a fiber decreases quadratically with decreasing radius).

Interest in the last decade in flexible electronics is driven by applications in consumer electronics (e.g. flexible laptop or smartphone screens), but also integration in the human body to design sensors that can be implanted on living organs 19. If bending flexibility is achieved by designing thin enough structures, stretching compliance is usually obtained through the use of wavy structures; in both case the relative rigidity of the material (silica) is dealt with by structural design. Some examples where the focus has been on the material side are the use of conductive polymers in the fabrication of conductive elastic materials 20]. Here we use in-drop coiling to design highly extensible fibers. Moreover we introduce the use of an auxiliary soft beam that helps in-drop coiling of these elastic fibers, and show that coiling can be achieved with electrically conductive materials.

The paper is organized as follows. In Section 2 we give thorough information on experimental methods and materials used. In Section 3 we recall the conditions under which an elastic fiber may coil inside a liquid drop. We then point out in Section 4 that gravity inevitably hinders the coiling procedure, but in Section 5 we introduce so the use of an auxiliary soft beam which on the contrary enhances this coiling procedure and enables us to show in Section 6 the in-drop coiling of a conductive relatively stiff fiber. We discuss our results in Section 7 and conclude. 


\section{Materials and Methods}

55

Different materials were used to fabricate the fibers in order to cover a large span of Young's moduli in the experiments. The thermoplastic polyether polyurethanes (TPU) fibers were fabricated from pellets (BASF) following a manual melt-spinning process described in 21] where $\mathrm{a}_{115}$ TPU pellet is molten at $225^{\circ} \mathrm{C}$ on a hot plate and then touched by the tip of needle. Pulling the needle rapidly then draws out a microfiber which immediately solidifies in the air, leaving the operator with a meter long microfiber. The different TPU's Young's moduli were mea- ${ }_{120}$ sured through a force displacement test using the FemtoTools micro-forces instrument FT-MTA02 (capacitive deflection measurement).

Polylactic acid (PLA) is commonly used for 3D printing, its Young's modulus was measured using a Shimadzu ${ }_{125}$ 70 AG-X Plus electromechanical test frame on a PLA wire of radius $1.5 \mathrm{~mm}$. Just as for the TPU, PLA fibers were fabricated with a melt-spinning technique: a small amount of PLA was molten on a hot plate $\left(290^{\circ} \mathrm{C}\right)$ and subsequently pulled out rapidly with the tip of a needle, thus creating ${ }_{130}$ 75 the solid microfiber.

Poly(3,4-ethylenedioxythiophene)poly(styrenesulfonate) (PEDOT:PSS, a conductive polymer) dry re-dispersible pellets from Sigma-Aldrich were used to draw fibers using the wetspinning process

80 described in 22, 23. A 2.9\%wt PEDOT:PSS-water ${ }_{135}$ solution was first prepared using a Mettler-Toledo MS scale. The solution was then injected through a calibrated needle (Adhesive Dispensing Ltd and Cluzeau Info Labo) out of a Terumo $10 \mathrm{~mL}$ glass syringe into an acetone bath.

85 As water is more soluble in acetone than in PEDOT:PSS, ${ }_{140}$ it flows out of the PEDOT:PSS, which itself is not soluble in acetone, and the injection leads to the contraction and solidification of a continuous flow of PEDOT:PSS. The resulting solid microfiber was then manually extracted

90 from the acetone bath and dried out in ambient air for ${ }_{145}$ about one minute. Even though the PEDOT:PSS fibers thereby created are not exactly the same as in 23], we use their value for the Young's modulus. Our PEDOT:PSS fibers showed a specific electric conductivity of $7 \mathrm{~S} / \mathrm{cm}$.

${ }_{95}$ End-to-end electrical resistance of straight and in-drop coiled PEDOT:PSS microfibers were measured using an ${ }_{150}$ ohmmeter (Amprobe 5XP-A). The electrical connections between macro-parts and the microfiber were established with a Hi-Bond Conductive Copper Tape.

No active control on the fiber's cross-section radius is possible with both of the aforementioned fabrication tech-155 niques, but it was optically measured after its fabrication for each fiber using a Leica macroscope (VZ85RC) with a Leica DFC-295 camera. The optical resolution of this macroscope is of the order of magnitude of the diameter of the thinnest fibers that were used (around 2 microns).160 Since the bending rigidity of a cylindrical fiber is proportional to the fourth power of the radius of its cross section, the fibers' radii are a key parameter in the experiments.
${ }_{110}$ Therefore an algorithm was written to take into account the blurriness of the image near the fiber edges, and after image post-processing the absolute uncertainty on the fiber's radii was lowered to $0.5 \mu \mathrm{m}$.

Zhermack Shore 8 Polyvinyl-siloxane (PVS), was used to fabricate the soft auxiliary beams. The following technique was used to obtain $\sim 2 \mathrm{~cm}$ long fibers with $\sim 100$ $\mu \mathrm{m}$ thin cross sections. Before reticulation, the PVS was poured on the $170 \mu \mathrm{m}$ wide side of a microscope glass slide (VWR, 22x50 mm, thickness No 1.5). A small part of the PVS then sat on the side when reticulation starts, whereas the surplus ran off the edges. After reticulation $(\approx$ 20 minutes at ambient temperature), a fiber is extracted from the side of the glass slide. Its cross section resembles a circular cap which we will here, for simplification, consider to be rectangular of height and width equal to those observed on the circular cap. These geometric dimensions were observed optically with the Leica macroscope. The aspect ratio $k=\frac{h_{\text {soft }}}{w_{\text {of }}}$ of the cross-section of the fiber was found to be $k=0.34$. The perimeter and the quadratic moment of inertia of the cross section were also approximated to be those of a rectangular cross section. Finally, the Young's modulus of our PVS was determined by a force-displacement measurement on a cylindrical fibre of radius $750 \mu \mathrm{m}$ and length $8.5 \mathrm{~cm}$.

The droplets used for the experiments are silicone oil droplets (viscosity $100 \mathrm{cSt}$ at $25^{\circ} \mathrm{C}$ ) from Sigma-Aldrich. It is to be mentioned that this silicone oil does not swell TPU, PLA and PEDOT:PSS, but does swell PVS. As a first order approximation, no Young's modulus rectifications were applied to the PVS beams. However, characteristic dimensions of these PVS beams increased by about $20 \%$ due to this swelling. Silicone oil was found to perfectly wet all of the used materials (Young-Dupré equilibrium wetting angle $\theta_{\mathrm{Y}}=0$ ) which is why we consider $\gamma_{\mathrm{SV}}-\gamma_{\mathrm{SL}}=\gamma \cos \theta_{\mathrm{Y}}=\gamma$, where $\gamma_{\mathrm{SV}}, \gamma_{\mathrm{SL}}$ and $\gamma$ are respectively the Solid-Vapor, Solid-Liquid and Liquid-Vapor specific interface energies.

In order to determine whether a fiber (with or without auxiliary soft fiber) was 'coilable' in a silicone oil droplet, about 10 droplets of different sizes were put on the fiber, seeking the best droplet size (big enough to allow coiling, but not too big for it not to be too heavy). If the fiber undergoes at least 2 coils inside one of the drops upon compression of its ends, at whichever hanging angle, the fiber is considered 'coilable'. If no droplet leads to this type coiling, the fiber is considered 'uncoilable'. Following this procedure, a fiber would first be tested alone (without auxiliary soft beam), and then would be affixed to the auxiliary soft beam and the composite fiber would be tested. The core fiber and the auxiliary beam stick to each other outside the droplet through the formation of a liquid bridge, but detach from one and other inside the liquid droplet. 


\begin{tabular}{lr} 
Material & Young's modulus $E$ \\
\hline PVS shore 8 & $200 \pm 30 \mathrm{kPa}$ \\
TPU E1170A & $9.50 \pm 2 \mathrm{MPa}$ \\
TPU E1185A & $23.6 \pm 4 \mathrm{MPa}$ \\
TPU E1198A & $66 \pm 10 \mathrm{MPa}$ \\
TPU E1174D & $560 \pm 40 \mathrm{MPa}$ \\
PEDOT:PSS & $2.5 \pm 0.7 \mathrm{GPa}$ \\
PLA & $2.7 \pm 0.7 \mathrm{GPa}$
\end{tabular}

Table 1: The materials used for fiber fabrication and their respective Young's modulus $E$.

\section{In-drop coiling of a bare fiber in the absence of gravity}

We consider a liquid drop sitting on an elastic fiber in the absence of gravity. The fiber has a circular cross-section of radius $a$, Young's modulus $E$, total length $L$. The liquid drop has total volume $\frac{4}{3} \pi R^{3}$. As long as the drop sits on a straight fiber, it adopts an unduloid shape [24, 25]. When $R \gg a$ the unduloid resembles a sphere (of radius $R$ ) and we will make this approximation even once the fiber is coiled inside the liquid drop. The drop-on-fiber system is held under tension $T$, see Fig. 1. Furthermore, as we consider small systems (typically $a \sim 10 \mu \mathrm{m}, R \sim 100 \mu \mathrm{m}$ ) we need to take surface forces into account. We note $\gamma_{\mathrm{SL}}$ the surface energy of the Solid-Liquid interface, $\gamma_{\mathrm{SV}}$ the surface energy of the Solid-Vapor interface, and $\gamma_{\mathrm{LV}}$ that of the Liquid-Vapor interface. Due to the stronger affinity of the fiber for the liquid than for the vapor (i.e. $\gamma_{\mathrm{SL}}<\gamma_{\mathrm{SV}}$ ), surface tension forces at the two menisci points $A$ and $B$ tend to compress the fiber inside the drop. If the compression is large enough, more fiber is reeled inside the drop and coiling occurs. We now compute the relation between the external tension $T$, surface tension forces, and other parameters, for the system to be in equilibrium. We list all the contributions to the total potential energy $\mathcal{V}$ of the system once in the coiling regime. First the surface energies are $2 \pi a \gamma_{\mathrm{SV}} L_{\mathrm{out}}, 2 \pi a \gamma_{\mathrm{SL}} L_{\text {in }}$ where $L_{\text {in }}$ is the length of the fiber inside the drop and $L_{\text {out }}$ that of the fiber outside the drop. As we do not allow the spherical drop to deform in this model, the surface energy $4 \pi R^{2} \gamma_{\mathrm{LV}}$ of the LiquidVapor interface stays constant and is therefore discarded. To compute the bending energy $\frac{1}{2} E I \int_{0}^{L_{\text {in }}} \kappa^{2}(s) \mathrm{d} s$ of the coiled fiber, where $I=\pi a^{4} / 4$ is the second moment of area of the circular cross-section of the fiber, we perform the simplification where the curvature $\kappa(s)$ of the fiber is uniform and equal to $1 / R$, with $s$ the arc length along the fiber. Finally we add the work of the external load $-T X$ where $X$ is the end-to-end distance of the system (here, $X \simeq L_{\text {out }}$ because $\left.2 R \ll X\right)$. We use $L_{\text {in }}+L_{\text {out }}=L$ to replace $L_{\text {in }}$ and find that, up to constant terms, the total potential energy of the system reads

$\mathcal{V}\left(L_{\text {out }}\right)=\left[2 \pi a\left(\gamma_{\mathrm{SV}}-\gamma_{\mathrm{SL}}\right)-\frac{1}{2} E I \frac{1}{R^{2}}-T\right] L_{\mathrm{out}}+$ const.

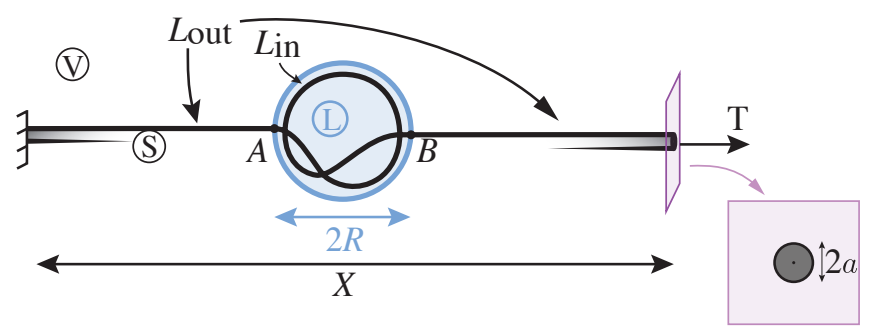

Figure 1: Cylindrical fiber of radius $a$ undergoing coiling inside a liquid drop of radius $R$ in absence of gravity. The fiber is held at a tension $T$.

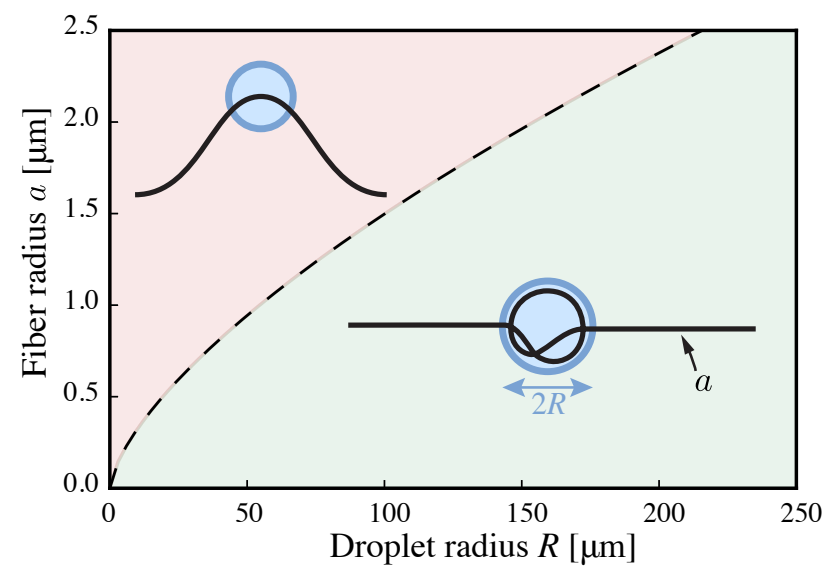

Figure 2: Graphical representation of eq. (3) for a silicone oil droplet $(\gamma=21 \mathrm{mN} / \mathrm{m}$ ) sitting on a bare fiber (of Young's modulus $E=1$ $\mathrm{GPa}$ in this example). For a given fiber radius $a$, in-drop coiling will be energetically favorable for a big enough drop. In this case, the compressive capillary force $2 \pi a \gamma$ overcomes the elastic bending force $\frac{\pi E a^{4}}{8 R^{2}}$ and the fiber is spooled inside the drop.

where we see that the linearity of $\mathcal{V}$ (Lout) implies that the load on the system, $T$, does not depend on its extension $X$, i.e. we have at equilibrium $\frac{\mathrm{d} \mathcal{V}}{\mathrm{d} L_{\mathrm{out}}}=0$

$$
T=2 \pi a \gamma-\frac{1}{2} E I \frac{1}{R^{2}}
$$

where we write $\gamma=\gamma_{\mathrm{SV}}-\gamma_{\mathrm{SL}}$. At first sight, one could then conclude that coiling inside the drop is always achievable (even when $\gamma_{\mathrm{SV}}<\gamma_{\mathrm{SL}}$ ) provided the right external load $T$ is applied. Nevertheless if $T$ is negative, that is if a global compression is applied on the system, then global buckling occurs at $T \propto-\frac{E I}{L^{2}}$, which is almost zero since $L$ is large. A necessary condition for coiling to occur in the absence of gravity is then $T>0$ in Eq. (2) or

$$
2 \pi a \gamma>\frac{\pi E a^{4}}{8 R^{2}}
$$

which yields a threshold for the fiber radius $a$ as function of $E, R$, and $\gamma$ with $a \propto R^{2 / 3} E^{-1 / 3}$. Fig. 2 shows this threshold between coilable and uncoilable systems for a TPU fiber and silicone oil droplet. Eq. (3) can be interpreted in terms of forces by saying that coiling is possible whenever 
the surface tension force of the drop $2 \pi a \gamma$ is larger than the bending reaction force $\frac{\pi E a^{4}}{8 R^{2}}$ of the fiber. We note that in the present case where gravity is absent, it is possible to coil virtually any fiber by choosing a large enough drop:

\section{In-drop coiling of a bare fiber in the gravity field}

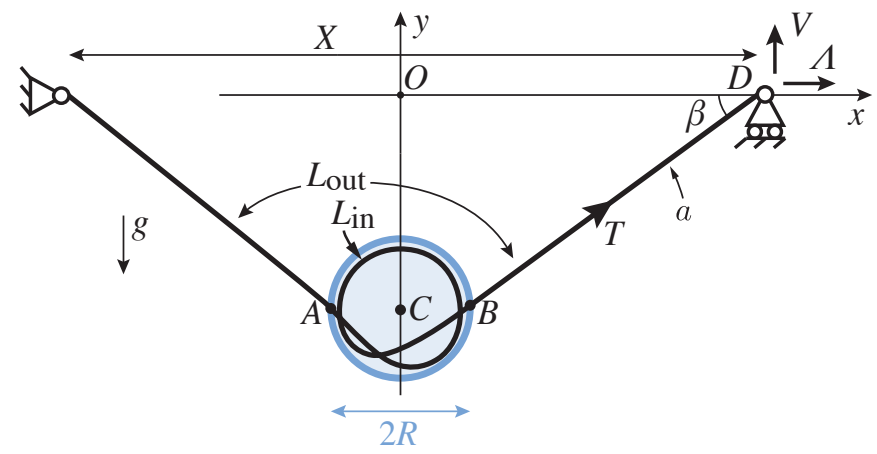

Figure 3: Cylindrical fiber of radius a undergoing coiling inside a liquid drop of radius $R$ in the gravity field. Due to gravity, the coiled state is sagging as the drop's weight pulls it downwards. The equilibrium state displays a sagging angle $\beta$ which depends on the system physical parameters, as described in equations 7 and 8 .

In the previous section we saw that, if gravity is neglected, coiling can be activated on virutally any fiber provided a large enough liquid drop is used. In the present section we show that once gravity is accounted for, there is an upper limit for the radius of the fiber (of a given material) for which coiling is possible. We consider the system of Fig. 3, write the total potential energy, and compute its equilibrium. The bending energy is $\frac{1}{2} E I \frac{1}{R^{2}} L_{\text {in }}$ and, as explained in the previous section, surface energies add up to $2 \pi \gamma L_{\text {out }}$ where $\gamma=\gamma_{\mathrm{SV}}-\gamma_{\mathrm{SL}}$. The potential energy for the weight of the drop is $m g y_{C}$ where $C$ is the center of the spherical drop and $m=\frac{4}{3} \pi \rho R^{3}, \rho$ being the density of the liquid and $g$ the acceleration of gravity. The work of the external load $\Lambda$ is $-\Lambda X$ where $X \approx L_{\text {out }} \cos \beta$. Using $L_{\text {in }}=L-L_{\text {out }}$, the total potential energy is then, up to constant terms

$\mathcal{V}\left(\beta, y_{C}, L_{\text {out }}\right)=\left(2 \pi \gamma-\frac{1}{2} \frac{E I}{R^{2}}-\Lambda \cos \beta\right) L_{\text {out }}+m g y_{C}$

The three variables of $\mathcal{V}$ are linked by the relation $2 y_{C}+$ $L_{\text {out }} \sin \beta=0$ which expresses the fact that point $D$ is constrained vertically. We therefore use

$$
\mathcal{L}=\mathcal{V}-V\left(2 y_{C}+L_{\text {out }} \sin \beta\right)
$$

to write the equilibrium of the system as $\left(\partial \mathcal{L} / \partial L_{\text {out }}, \partial \mathcal{L} / \partial \beta, \partial \mathcal{L} / \partial y_{C}\right)=\mathbf{0}$. Here $V$ is the Lagrange multiplier associated with the constraint in the vertical position of $D$ and is therefore interpreted as

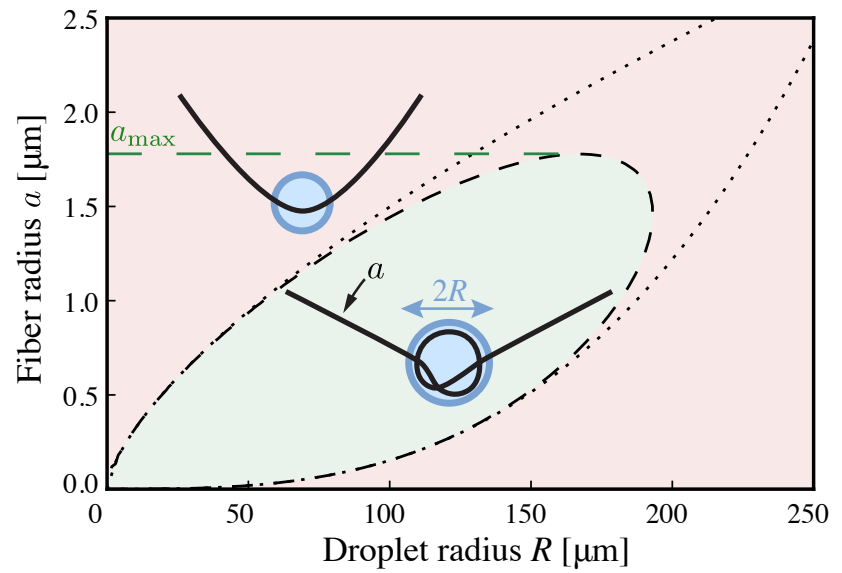

Figure 4: Graphical representation of the inequality (9) for a silicone oil drop $\left(\gamma=21 \mathrm{mN} / \mathrm{m}, \rho g=9600 \mathrm{~N} / \mathrm{m}^{3}\right)$ sitting on a fiber (Young's modulus $E=1 \mathrm{GPa}$ in this example). For a given fiber of radius $a$ to undergo in-drop coiling, the drop radius $R$ has to be large enough. However, the drop's weight will hinder coiling and eventually prevent it when $R$ exceeds a certain treshold. This generates a coiling phase "tongue" which displays a limit for the fiber radius $a_{\max }=a_{\max }(\gamma, \rho g, E)$ described in equation 10 . The upper dotted curve corresponds to the elasto-capillary limit described in section 3 (no gravity) and the lower dotted curve shows the biggest drop that can sit on a given fiber without falling (gravito-capillary limit $\left.4 / 3 \pi \rho g R^{3}<2 \times 2 \pi a \gamma\right)$ [].

the vertical reaction force from the support at the right extremity of the fiber. Equilibrium is such that

$$
\begin{aligned}
\Lambda \cos \beta+V \sin \beta & =2 \pi a \gamma-\frac{1}{2} \frac{E I}{R^{2}} \\
V & =\frac{m g}{2} \\
V \cos \beta & =\Lambda \sin \beta
\end{aligned}
$$

The last equation shows that the total external force on the system at point $D, \Lambda \boldsymbol{e}_{\boldsymbol{x}}+V \boldsymbol{e}_{\boldsymbol{y}}$, is along the direction $\beta$, that is the fiber outside the drop carries no shear force but a tension $T=\Lambda \cos \beta+V \sin \beta$. The first and second equations in $(6)$ are then rewritten as

$$
\begin{aligned}
T & =2 \pi a \gamma-\frac{1}{2} \frac{E I}{R^{2}} \\
m g & =2 T \sin \beta
\end{aligned}
$$

where (8) shows that the tension $T$ has to be positive, a limitation already found in the previous Section. Moreover once the parameters $m g, a, \gamma, E I, R$ are given, (7)-(8) yield the angle $\beta$ at which the system is going to experience coiling, the equilibrium angle $\beta$ increasing with the weight $m g$. As $\beta<\frac{\pi}{2}$ a necessary condition for coiling to occur in the presence of gravity is $T>\frac{m g}{2}$ which can be rewritten as

$$
2 \pi a \gamma>\frac{\pi E a^{4}}{8 R^{2}}+\frac{2}{3} \pi \rho g R^{3}
$$

In the force interpretation introduced in the previous Section, this means that for coiling to be possible, the surface 


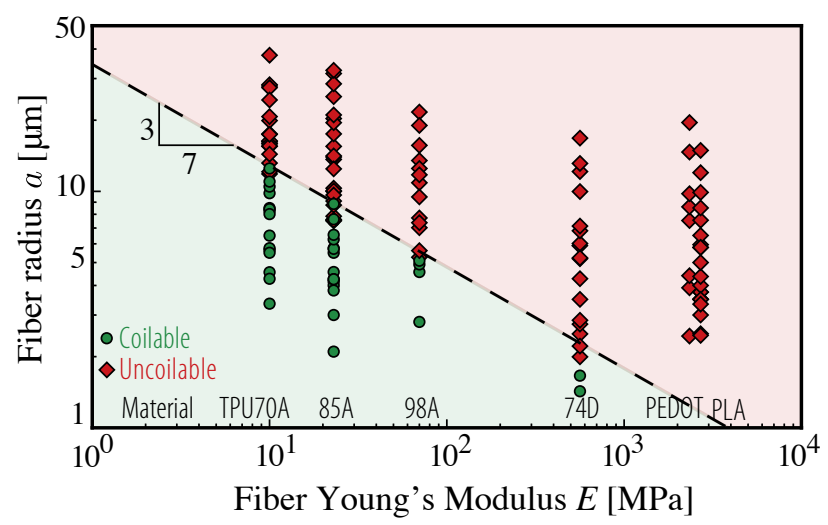

Figure 5: Theoretical and experimental coilable and uncoilable fibers of different materials in the corresponding optimal silicone oil droplet. The theoretical limit between the coilable and uncoilable systems is given in equation 10 and experiments validate the theoretical power law $a_{\max } \propto E^{-3 / 7}$ and the corresponding prefactor. For PEDOT:PSS and PLA fibers, no coiling ever occurred as we were not able to produce thin enough fibers. $\gamma=21 \mathrm{mN} / \mathrm{m}, \rho g=9600$ $\mathrm{N} / \mathrm{m}^{3}$.

tension has to overcome the weight of the drop in addition to the bending force of the fiber. Fig. 4 shows the region, in the plane $(a, R)$, where coiling is possible. We see that in the presence of gravity, once the material parameters $\rho g, \gamma, E$ are given, there is a maximum value $a_{\max }$ to the radius $a$ of the fiber for which coiling is possible. Differentiating 9 with regard to $R$, we compute this maximum radius to be

$$
a_{\max }=2.78 \gamma^{5 / 7}(\rho g)^{-2 / 7} E^{-3 / 7}
$$

This limitation is due to the fact that exceedingly large drops cannot fit on a fiber, as explained in [7]. For liquid parameters (used throughout the present paper) $\rho g=9600$ $\mathrm{N} / \mathrm{m}^{3}$ and $\gamma=21 \mathrm{mN} / \mathrm{m}$, we plot in Fig. 5 the radius $a_{\max }$ as a function of the Young's modulus $E$ of the material of the fiber. In the same figure we report on the experimental coiling character of several fibers and find a good agreement with theory.

\section{Coiling the uncoilable: the auxiliary soft beam}

The elasto-capillary coiling mechanism allows to create composite threads that are highly extensible: as a large amount of fiber may be spooled inside a droplet, the total length $X$ of the system may vary by more than a factor ten [14. This extreme mechanical feature could be of interest to design components for stretchable electronics devices. Typical Young's modulus for conducting materials are $\sim 100 \mathrm{GPa}$, which requires the use of sub-micronic fibers, see Fig. 5 and Eq. (10). Equation (9) shows that for coiling to occur, surface tension as to overcome the sum of the weight of the drop and the bending force of the fiber. A large Young's modulus $E$ implies a large bending force

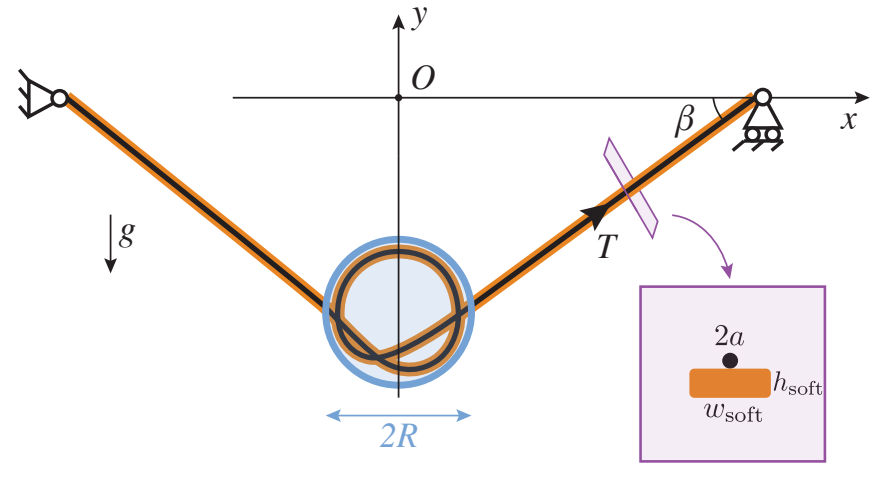

Figure 6: Cylindrical fiber of radius $a$ affixed to a soft beam (rectangular cross section: height $h_{\text {soft }}$, width $w_{\text {soft }}$ ) undergoing coiling inside a liquid drop of radius $R$ in the gravity field. Due to gravity, the coiled state is sagging and the equilibrium state displays a sagging angle $\beta$ which depends on the system physical parameters. The composite fiber (core fiber + soft beam) inherits the large beam's perimeter but its bending stiffness mainly depends on that of the core fiber.

unless we use a drop with a large radius $R$. Nevertheless we have seen in the previous section that increasing the drop radius is eventually unfavorable to coiling, as it makes the weight become too large. Alternatively we can try to increase the surface tension force $2 \pi a \gamma$. The span of the possible values for $\gamma$ being restricted for classical liquids, we are left with the option of increasing the contour length of the triple line, that is the perimeter $2 \pi a$ of the fiber. As increasing a rapidly increases the bending force we seem to be in an inextricable situation. Yet the use of a second fiber with low Young's modulus and large perimeter is a way out. We use the system of Fig. 6 where our fiber of circular cross-section (radius $a$ ) is combined to a beam of rectangular cross-section (width $w_{\text {soft }}$, height $h_{\text {soft }}$ ) and made of soft material (Young's modulus $E_{\text {soft }}$ ). The fiber adheres to the beam when they stand in the air but they separate once they are in the drop. On first approximation we consider the difference $\gamma=\gamma_{\mathrm{SV}}-\gamma_{\mathrm{SL}}$ to have the same value for both the beam and the fiber. Moreover we neglect the area of the fiber compared to that of the beam ( $\left.a \ll h_{\text {soft }}, w_{\text {soft }}\right)$. The total potential energy of the system is then written as

$$
\begin{aligned}
\mathcal{V}=\left[2 \gamma\left(h_{\mathrm{soft}}+w_{\mathrm{soft}}\right)-\Lambda \cos \beta-\right. \\
\left.\frac{1}{2 R^{2}}\left(E I+E_{\mathrm{soft}} I_{\text {soft }}\right)\right] L_{\mathrm{out}}+m g y_{C}
\end{aligned}
$$

which we minimize following the same procedure as in the previous section. The condition for the combined fiberbeam system to coil is then

$$
2 \gamma\left(h_{\mathrm{soft}}+w_{\mathrm{soft}}\right)>\frac{1}{2 R^{2}}\left(E I+E_{\mathrm{soft}} I_{\mathrm{soft}}\right)+\frac{2}{3} \pi \rho g R^{3}
$$

with $I_{\text {soft }}=\frac{1}{12} w_{\text {soft }} h_{\text {soft }}^{3}$. The region determined by this inequality is plotted in Fig. 7 where it is compared to the coiling region for the bare fiber. 


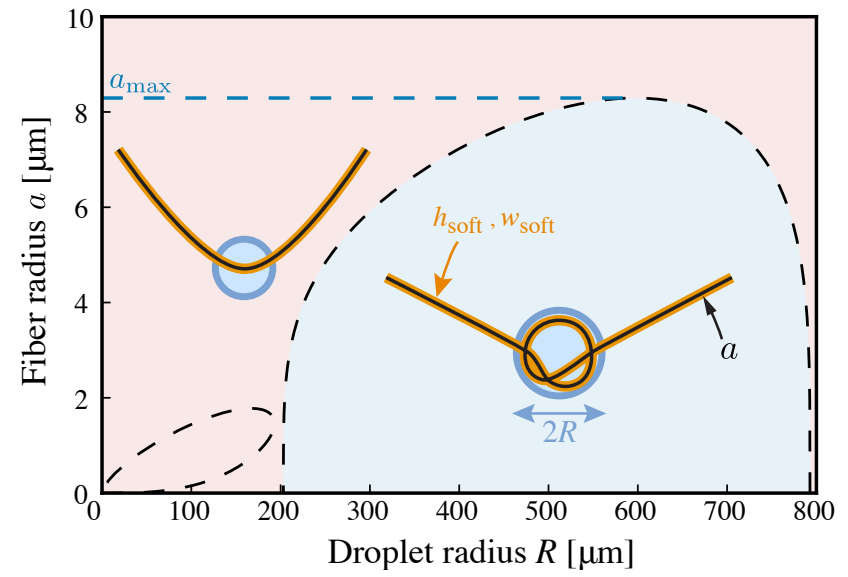

Figure 7: Graphical representation of the inequality 12 (blue210 tongue) for a silicone oil drop $\left(\gamma=21 \mathrm{mN} / \mathrm{m}, \rho g=9600 \mathrm{~N} / \mathrm{m}^{3}\right)$ sitting on a composite fiber composed of a core fiber of radius $a$ and Young's modulus $E=1 \mathrm{GPa}$ in this example, and a soft auxiliary PVS beam $\left(w_{\text {soft }}=190 \mu \mathrm{m}, h_{\text {soft }}=65 \mu \mathrm{m}, E_{\text {soft }}=200 \mathrm{kPa}\right)$. The small tongue near the origin corresponds to the one shown in figure 4. for a silicone oil droplet sitting on a bare fiber. The soft auxiliary ${ }_{215}$ artifact allows to achieve coiling for much thicker fibers (larger $a$ ). The largest theoretical coilable fiber has a radius $a_{\max }$ explicited in equation 13 .

We note that the soft beam brings the possibility to use bigger drops and hence coil thicker (i.e. larger $a$ ) fibers. As in the previous Section, bearing in mind that $E I=\frac{\pi E a^{4}}{4}, 220$ we calculate from 12 the maximum value $a_{\max }$ for the radius of the fiber for which coiling occurs in the combined system:

$$
\begin{aligned}
a_{\max }=E^{-1 / 4}\left(1 . 6 2 \left(\gamma \left(h_{\mathrm{soft}}\right.\right.\right. & \left.\left.+w_{\mathrm{soft}}\right)\right)^{5 / 3}(\rho g)^{-2 / 3} \\
& \left.-1.27 E_{\mathrm{soft}} I_{\text {soft }}\right)^{1 / 4}
\end{aligned}
$$

Fixing $\rho g$ and $\gamma$, we study how $a_{\max }$ varies with the beam parameters for $h_{\text {soft }}=k w_{\text {soft }}$. We first optimize the cross-230 section and find that $a_{\max }$ is maximum for

$$
w_{\mathrm{soft}}=f(k) \gamma^{5 / 7}(\rho g)^{-2 / 7} E_{\mathrm{soft}}^{-3 / 7}
$$

see Fig. 9-left. This optimum value for $w_{\text {soft }}$ and the max-235 imum value of $a_{\max }$ are plotted in Fig. 9 -bottom as a function of $E_{\text {soft }}$. We can now inject this optimum value 14 in (13) to obtain a maximum radius value that only depends on material parameters

$$
\max \left(a_{\max }\right)=g(k) E^{-1 / 4} \gamma^{5 / 7}(\rho g)^{-2 / 7} E_{\text {soft }}^{-5 / 28}
$$

In our experiments, the thickness to width ratio $k=\frac{h_{\text {soft }}}{w_{\text {soft }}}$ of the auxiliary beam is $k=0.34$. For readability, the dependence on $k$ of $w_{\text {soft }}$ in eq. 14 and $\max \left(a_{\max }\right)$ in eq. 15,45 is given by two functions $f(k)$ and $g(k)$ who naturally derive from the optimization calculations, they respectively take values of $f(0.34)=10.9$ and $g(0.34)=3.0$.
Experimentally we use a beam with $E_{\text {soft }}=200 \mathrm{kPa}$, $h_{\text {soft }}=65 \mu \mathrm{m}$, and $w_{\text {soft }}=190 \mu \mathrm{m}$ not far from the optimum value, see Fig. 9-top. We then study how $a_{\text {max }}$ varies as the Young's modulus $E$ of the fiber material is varied, see Fig. 8. For the combined system $a_{\max } \propto E^{-1 / 4}$, which is favorable compared to the $E^{-3 / 7}$ behaviour observed in the case of a bare fiber. We find a good agreement for the power law of $a_{\max }$ as a function of $E$, but a $\times 2$ systematic error on the prefactor between theory and experiments for fibers with modulus in the MPa-GPa interval. The presented model considers that for coiling to happen, the surface tension force has to overcome the weight of the drop plus the elastic bending force of the fiber. This bending force is deduced from the curvature of a spherically spooled elastic fiber. However, at early stage of buckling, the elastic bending force is higher than that of the post-buckled (i.e. spooled) fiber. Moreover, experimentally, we observe that the the composite fiber buckles in mode 2 (i.e. with an "S" shape), therefore increasing the difference between the modeled bending force, and the real one. This may explain the constant $\times 2$ error between prediction and experiment.

\section{Towards electronic functionalisation : coiling conductive PEDOT:PSS fibers}

As mentioned earlier, the coiling of a microfiber inside a droplet is a building block for highly extensible threads. Moreover, in-drop coiling stores the fiber surplus and releases it while keeping the system under tension and taut. This feature could be of interest for stretchable electronics where extensible electric connections are sought. As shown in figure 8, the auxiliary soft fiber technique allows for micronic fibers to undergo in-drop coiling for materials with Young's moduli up to few GPa, whereas they would have to be sub-micronic in the case of a bare fiber.

PEDOT:PSS is a conductive GPa-modulus polymer with which micronic fibers can easily be spun. A single 3.5 centimeter long PEDOT:PSS fiber spun with a $50 \mu \mathrm{m}$ needle has a cross section with radius $a_{\mathrm{PEDOT}}=4.2 \mu \mathrm{m}$ and an end-to-end resistance of about $Z_{0}=0.90 \mathrm{M} \Omega$. This corresponds to a specific conductivity of $\sigma=7 \mathrm{~S} / \mathrm{cm}$, which is approximately what is found for the P-grade PEDOT:PSS in [23], $\sigma=11 \mathrm{~S} / \mathrm{cm}$. Figure 10 shows the endto-end electrical resistance $Z(X)$ of this conductive fiber coupled with a PVS auxiliary beam $\left(w_{\text {soft }}=190 \mu \mathrm{m}\right.$ and $\left.h_{\text {soft }}=65 \mu \mathrm{m}\right)$, as a function of the end-to-end distance $X$. Starting with $X=2 R=1 \mathrm{~mm}$ where the entire fiber and beam are coiled inside the drop, we stretch the system up to a factor 20 where everything is straight. Throughout this $1900 \%$ extension the end to end resistance of the composite system does not decrease but fluctuates around the nominal resistance $Z_{0}$. We then design a second system where 2 PEDOT:PSS fibers are coupled to the PVS auxiliary beam and found a weakly fluctuating resistance around $50 \%$ of $Z_{0}$. We conclude that no permanent short 
circuits happen in spite of the multiple coils contacting inthe droplet. However, brief spontaneous short-circuits could explain the observed fluctuations in resistance. A one-loop length shortening (i.e. $2 \pi R$ ) would lead to a $9 \%$ drop of end to end resistance, consistant with the typically observed fluctuations of figure 10 A $\left(2 R=1 \mathrm{~mm}\right.$ and the ${ }^{305}$ total probed length is $3.5 \mathrm{~cm}$ ).

\section{Discussion}

In conclusion, we showed that by introducing a soft supporting beam we could induce in-drop coiling on fibers with an a priori too large bending rigidity: the beam-fiber composite system coils in situations where the bare fiber would not. We thus have designed a composite system ca- ${ }^{315}$ pable of coping with extension ratios larger than twenty while staying functional. The high extensibility is due to the liquid drop that stores and gives back excess length, but liquid forces also enable the system to stay taut. The ${ }^{320}$ conductor property is brought by a fiber that needs an auxiliary supporting beam to coil inside the drop. Like in every successful hybrid system, each component brings its own function while gently combining with the others. ${ }^{325}$

270 The mechanical response of the composite system is inherited from that of its components. A bare conductive fiber, of length $L$, carrying a drop does not coil and hence exhibits a force-extension curve shown in red in Fig. 11 . $^{330}$ if the end-to-end distance $X$ is larger than $L$, the fiber is stretched and the applied tension $T$ grows linearly with a steep slope, due to the high Young's modulus of the fiber, while if $X<L$ global buckling occurs and the applied tension is negative. Now if one considers the soft supporting beam alone, stretching $(X>L)$ is achieved with a gentle slope and compression $(X<L)$ leads to in-drop coiling at a constant plateau value $T_{p}$, see the orange curve in Fig. 11. The composite system inherits from the behavior of the soft supporting beam under compression, with an in-drop coiling at the same plateau value $T_{p}$, and from the behavior of the conductive fiber under extension with the same steep slope when $X>L$, see the blue curve in Fig. 11 .

We further showed that the composite system is con- ${ }_{350}$ ducting electricity with a constant resistance over a large range of end-to-end distances (from $X=L / 20$ to $X=L$ ): in-drop coiling does not affect the conductive character of the fiber. Finally we gave the limiting radius of the fiber $_{355}$ for which in-drop coiling could be achieved, as a function of material properties (Young's moduli $E$ and $E_{\text {soft }}$ ) and liquid properties (density $\rho g$ and surface tension $\gamma$ ). Further work involves the study of other geometries for the sup- 360 porting beam (e.g. cylindrical coating) and other physical properties for the functional fiber (e.g. light conductivity) with the aim to design super-extensible conductive

\section{Acknowledgments}

S.N. gratefully thanks F.-O. Lende for continuous support and motivation. We also thank Natacha Krins and Régis Wunenburger for their precious experimental help and advices. The present work was supported by ANR grants ANR-09-JCJC-0022-01, ANR-14-CE07-002301, and ANR-13-JS09-0009. Financial support from 'La Ville de Paris - Programme Émergence' and from the CNRS (PEPS-PTI grant) is also gratefully acknowledged, along with travel support from the Royal Society, through the International Exchanges Scheme (grant IE130506).

\section{References}

[1] A. E. Cohen, L. Mahadevan, Kinks, rings, and rackets in filamentous structures, Proceedings of the National Academy of Sciences of the United States of America 100 (21) (2003) 1214112146. doi:10.1073/pnas.1534600100

[2] B. Roman, J. Bico, Elasto-capillarity: deforming an elastic structure with a liquid droplet, Journal of Physics: Condensed Matter 22 (49) (2010) 493101. doi:10.1088/0953-8984/22/49/ 493101.

[3] R. Syms, E. Yeatman, V. Bright, G. Whitesides, Surface tension-powered self-assembly of microstructures - the stateof-the-art, Journal of Microelectromechanical Systems 12 (4) (2003) 387-417. doi:10.1109/JMEMS.2003.811724

[4] A. Antkowiak, B. Audoly, C. Josserand, S. Neukirch, M. Rivetti, Instant fabrication and selection of folded structures using drop impact, Proceedings of the National Academy of Sciences 108 (26) (2011) 10400-10404. doi:10.1073/pnas.1101738108

[5] B. Andreotti, O. Baumchen, F. Boulogne, K. E. Daniels, E. R. Dufresne, H. Perrin, T. Salez, J. H. Snoeijer, R. W. Style, Solid capillarity: when and how does surface tension deform soft solids?, Soft Matter 12 (2016) 2993-2996. doi:10.1039/ C5SM03140K

[6] J. Bico, B. Roman, L. Moulin, A. Boudaoud, Adhesion: Elastocapillary coalescence in wet hair, Nature 432 (7018) (2004) 690-690. doi:10.1038/432690a

[7] É. Lorenceau, C. Clanet, D. Quéré, Capturing drops with a thin fiber, Journal of Colloid and Interface Science 279 (1) (2004) 192-197. doi:10.1016/j.jcis.2004.06.054

[8] C. Duprat, S. Protiere, A. Y. Beebe, H. A. Stone, Wetting of flexible fibre arrays, Nature 482 (7386) (2012) 510-513. doi: $10.1038 /$ nature 10779

[9] A. Sauret, A. D. Bick, C. Duprat, H. A. Stone, Wetting of crossed fibers: Multiple steady states and symmetry breaking, EPL (Europhysics Letters) 105 (5) (2014) 56006. doi:10.1209/ 0295-5075/105/56006

[10] F. Vollrath, D. T. Edmonds, Modulation of the mechanical properties of spider silk by coating with water, Nature 340 (1989) 305-307.

[11] S. Jung, C. Clanet, J. W. M. Bush, Capillary instability on an elastic helix, Soft Matter 10 (18) (2014) 3225-3228. doi: 10.1039/C3SM52629A

[12] F. Vollrath, Spider webs and silks., Scientific American 266 (3) (1992) 70-76.

[13] R. D. Schulman, A. Porat, K. Charlesworth, A. Fortais, T. Salez, E. Raphaël, K. Dalnoki-Veress, Elastocapillary bending of microfibers around liquid droplets, Soft matter 13 (4) (2017) 720-724. doi:10.1039/C6SM02095J

[14] H. Elettro, S. Neukirch, F. Vollrath, A. Antkowiak, In-drop capillary spooling of spider capture thread inspires hybrid fibers with mixed solid-liquid mechanical properties, Proceedings of the National Academy of Sciences of the USA 113 (22) (2016) 6143-6147. doi:10.1073/pnas.1602451113

[15] H. Elettro, F. Vollrath, A. Antkowiak, S. Neukirch, Coiling of an elastic beam inside a disk: A model for spider-capture silk, 
International Journal of Non-Linear Mechanics 75 (2015) 59-66. doi:10.1016/j.ijnonlinmec. 2015.03.008

[16] M. Ashby, Y. Bréchet, Designing hybrid materials, Acta Materialia 51 (19) (2003) 5801-5821. doi:10.1016/S1359-6454(03) 00441-5

[17] A. Greiner, J. H. Wendorff, Electrospinning: A fascinating method for the preparation of ultrathin fibers, Angewandte Chemie International Edition 46 (30) (2007) 5670-5703. doi: 10.1002/anie.200604646

18] J. A. Kluge, O. Rabotyagova, G. G. Leisk, D. L. Kaplan, Spider silks and their applications, Trends in Biotechnology 26 (5) (2008) 244-251. doi:10.1016/j.tibtech.2008.02.006

[19] J. A. Rogers, T. Someya, Y. Huang, Materials and mechanics for stretchable electronics, Science 327 (5973) (2010) 1603-1607. doi:10.1126/science.1182383

[20] T. S. Hansen, K. West, O. Hassager, N. B. Larsen, Highly stretchable and conductive polymer material made from poly (3, 4-ethylenedioxythiophene) and polyurethane elastomers, Advanced functional materials 17 (16) (2007) 3069-3073. doi: $10.1002 / \mathrm{adfm} .200601243$

[21] H. Elettro, Elasto-capillary windlass: from spider silk to smart actuators, Ph.D. thesis, Université Pierre et Marie Curie-Paris VI (2015)

[22] H. Okuzaki, M. Ishihara, Spinning and characterization of conducting microfibers, Macromolecular Rapid Communications 24 (3) (2003) 261-264. doi:10.1002/marc.200390038

[23] H. Okuzaki, Y. Harashina, H. Yan, Highly conductive PEDOT/PSS microfibers fabricated by wet-spinning and diptreatment in ethylene glycol, European Polymer Journal 45 (1) (2009) 256-261. doi:10.1016/j . eurpolymj . 2008.10.027

24] H. Poincaré, Capillarité, Jacques Gabay, 1895.

[25] B. J. Carroll, The accurate measurement of contact angle, phase contact areas, drop volume, and laplace excess pressure in dropon-fiber systems, Journal of Colloid and Interface Science 57 (3)
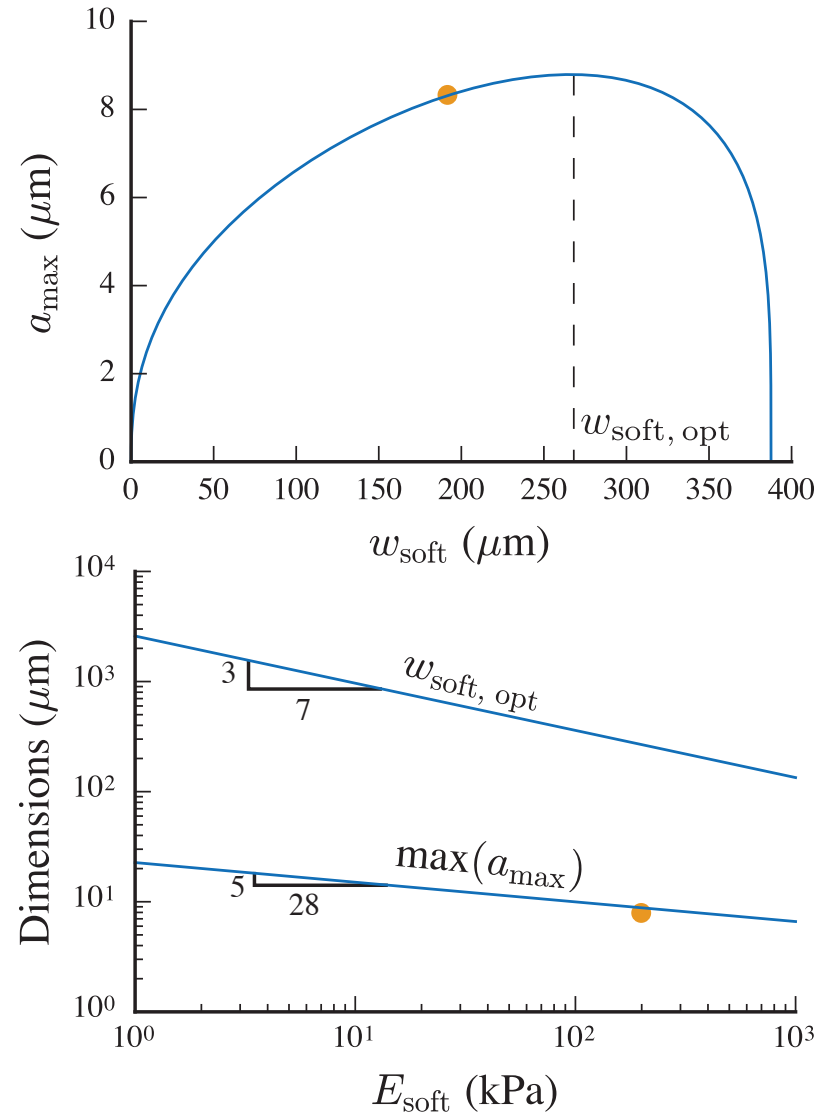

Figure 9: Optimal dimensions for the fiber and the auxiliary beam width. The auxiliary beam has Young's modulus $E_{\text {soft }}$ (PVS) and an aspect ratio $k=h_{\mathrm{soft}} / w_{\mathrm{soft}}=0.34$ (value used experimentally). The silicone oil drop has $\gamma=21 \mathrm{mN} / \mathrm{m}$ and $\rho g=9600 \mathrm{~N} / \mathrm{m}^{3}$. The core fiber in this example has a Young's modulus of $E=1 \mathrm{GPa}$. Top: Largest coilable fiber radius $a_{\max }$ as a function of the width $w_{\text {soft }}$ of the auxiliary beam of Young's modulus $E_{\text {soft }}=200 \mathrm{kPa}$ as given by equation (13). The function has a maximum at $w_{\text {soft }}=w_{\text {soft, opt }}$, see 14. The orange dot corresponds to the experimentally-used auxiliary beam, which was not far from being optimal. Bottom: $w_{\text {soft, opt }}$ as a function of the Young's modulus $E_{\mathrm{soft}}$ of the auxiliary beam, equation (14, and the corresponding maximum coilable core fiber's radius $\max \left(a_{\max }\right)$, equation 15 . The orange dot shows the value $a_{\max }$, given by equation 13 , for the experimentally used auxiliary beam. 


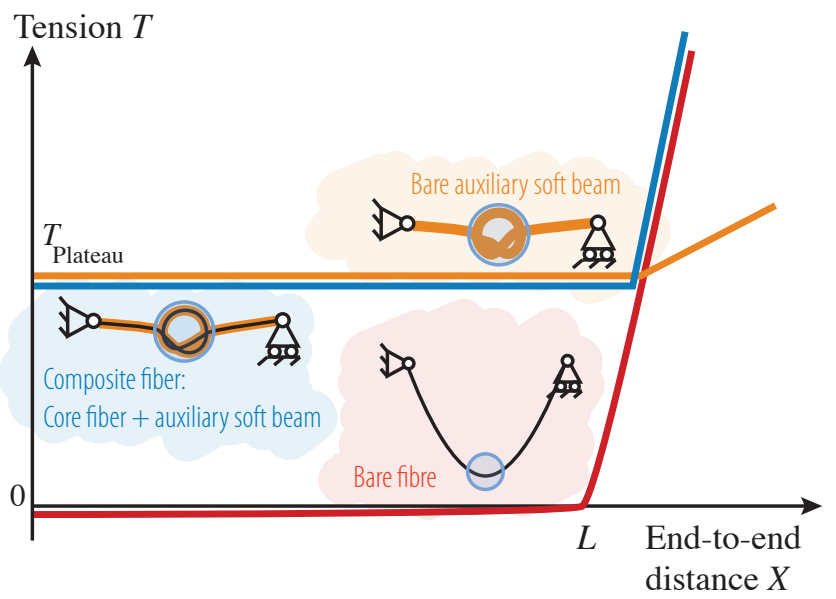

Figure 11: Phenomenological mechanical behaviours for the different systems. 

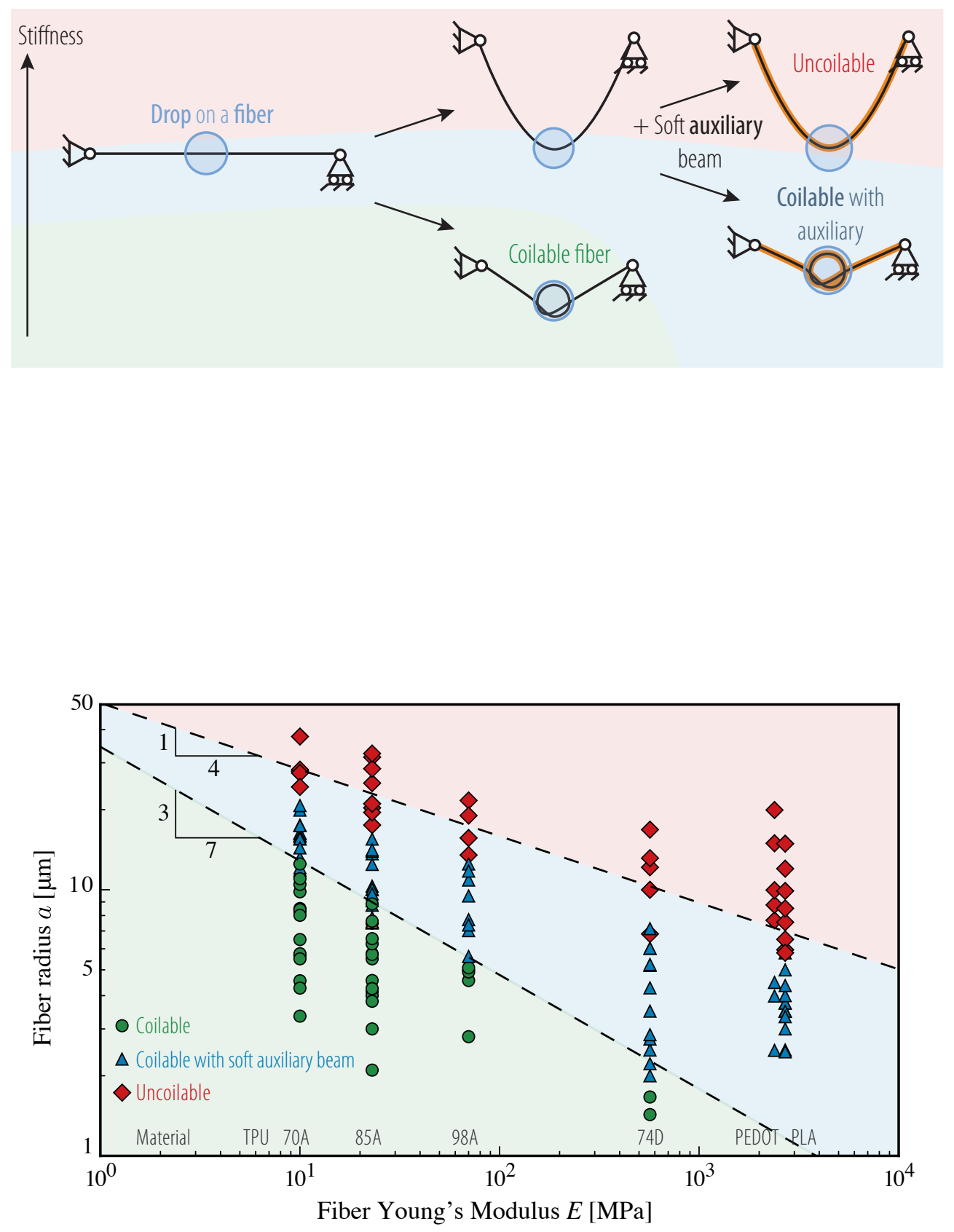

Figure 8: Theoretical and experimental coilable and uncoilable fibers of different materials in the corresponding optimal silicone oil droplet $\left(\gamma=21 \mathrm{mN} / \mathrm{m}, \rho g=9600 \mathrm{~N} / \mathrm{m}^{3}\right)$. Composite fibers are composed of a core fiber (radius $a$ and Young's modulus $E$ ) and an auxiliary PVS beam $\left(w_{\text {soft }}=190 \mu \mathrm{m}, h_{\text {soft }}=65 \mu \mathrm{m}, E_{\text {soft }}=200 \mathrm{kPa}\right)$. The theoretical limit between the coilable bare fiber and the coilable fiber with auxiliary beam is given by equation (10). The theoretical limit between coilable fibers with auxiliary beam and uncoilable fibers is given by equation 15. For PEDOT:PSS and PLA fibers, no coiling could happen for a bare fiber but thanks to the auxiliary beam strategy, micronic fibers were coiled inside silicone oil droplets. The theoretical power law $a_{\max } \propto E^{-1 / 4}$ seems appropriate to describe the frontier between the coilable with auxiliary beam and the uncoilable region of this phases diagram. However, a systematic error of about a factor 2 between theory and experiments shows that this simple model could be refined. 


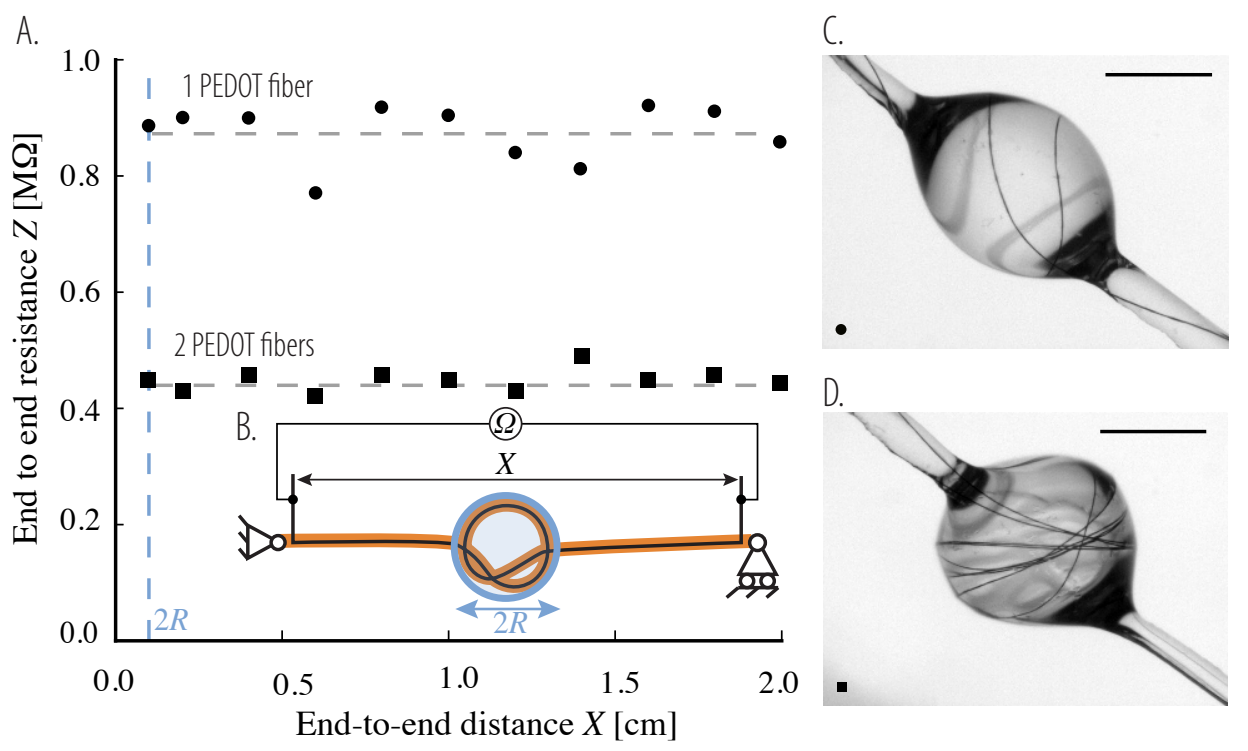

Figure 10: Coiling conductive micronic PEDOT:PSS fibers $\left(a_{\text {PEDOT }}=4.2 \mu \mathrm{m}\right)$ inside a silicone oil droplet with the assistance of a soft auxiliary beam. This composite fiber conducts electricity throughout the $1900 \%$ stretching test. (A). Electrical resistance versus of end-to-end distance $X$ of the fiber for 1 and 2 PEDOT:PSS fibers and one soft auxiliary PVS beam. The resistance was measured between two points, further apart than the actual ends of the auxiliary beam (total fiber length: $3.5 \mathrm{~cm}$ ), see (B). Pictures (C) and (D) show macroscope snapshots of the droplets for $X=1.6 \mathrm{~cm}$ and $X=0.8 \mathrm{~cm}$ for 1 and 2 PEDOT:PSS fibers respectively. The dark PEDOT:PSS can easily be spotted inside and outside the droplet. The PVS beam, however, is only visible outside the droplet due to a refraction index too close from that of the silicone oil. Scale bar on both pictures: $500 \mu \mathrm{m}$. 